\title{
IUD perforation and embedment within omentum: a rare and perplexing incidence
}

\author{
azam tarafdari ${ }^{1}$, mahrooz malek $^{1}$, elaheh pahlavan falahy ${ }^{1}$, and Alireza hadizadeh ${ }^{1}$ \\ ${ }^{1}$ Tehran University of Medical Sciences
}

December 8, 2021

\begin{abstract}
intrauterine rarely fail which results in pregnancy. Meanwhile, these devices can perforate uterine and migrate through abdomen. Our case experienced IUD failure and perforation simultaneously and the device was embedded in omentum and shifted rapidly which made it hard to localize and could only be removed using ultrasonography guidance
\end{abstract}

\section{Title}

IUD perforation and embedment within omentum: a rare and perplexing incidence

\section{Keywords}

IUD, uterine perforation, IUD failure, omentum, ultrasonography

\section{Introduction}

intrauterine devices (IUD) are one of the most frequently used methods of contraception. Even though IUDs are effective, they can result in failure. The studies show that there is no difference between different types of IUDs in regards to complications and failure. [1-3] [4] complications include a wide range, from failures in insertion and perforation to syncope and bradycardia. since these complications are unpredictable, a clinician must always be vigilant in regards to management of adverse outcomes. [5]

IUD failures can result in pregnancy, this incidence is particularly important since they can lead to ectopic pregnancy, preterm labor and miscarriage.[1 26 ] Meanwhile, IUDs are also responsible for uterine perforation. This incidence though rare and uncommon, happens mostly during the post-partum period. [3 7]An IUD might fully or partially penetrate the uterine; as described by the Zakin et al, the most common location for a complete perforation is the pouch of Douglas.[8] It can also attach either loosely or tightly to omentum. On a rare occasion these devices can become embedded in myometrium. other cases have also reported that IUDs can migrate within abdominal cavity and can potentially cause perforations.

Although uterine perforation might even cause peritonitis and it is a potentially life-threatening incidence, it is a very rare incidence and most of times it is asymptomatic but it can manifest symptoms like abnormal uterine bleeding and pain. [9] [8] [4] [10] [11] Several imaging methods have been utilized for diagnosis but the most frequently used methods are simple pelvic radiographs and transvaginal sonography. [5] [12] [13] It has been suggested that TVS is a more accurate modality in respect to diagnosis and localization. [12 14] [5]In a previous case series, it was reported that the devices were localized with CT-scan since TVS had missed the devices particularly when the had migrated in upper abdomen. [9] [13]Depending on the location of IUD, the techniques for removal can differ and can sometimes be challenging. [12] When these devices cannot be removed at the office or when their respective location indicates surgical removal, laparoscopic surgery is the preferred method, although novel studies suggest that removal is not warranted when the patient is not 
symptomatic as the risks of surgery are not justifiable. [15] [5 14] [6]Deeply perforated devices can also lodge into different organs within the abdominopelvic cavity; however, the most common site is the omentum. [16] [17] [13] [10] [6] [18]

In this article, we report a 30-year-old woman on her third gravidity who had received IUD insertion 3 months prior to pregnancy and in her following work up the IUD wasn't localized within uterine cavity. the localization and surgical removal were very challenging since the IUD was completely embedded within omentum.

\section{Case presentation}

A 30-year-old woman who, based on last menstrual period, was 7 weeks and 4 days pregnant, was presented to office while complaining of abdominal pain. She stated that 3 months prior to the date she had a copper 380t IUD insertion. 2 months past insertion, in a work-up following menstrual retardation it was revealed that the patient was actually pregnant. The patient underwent ultrasonographic imaging; the live embryo with cardiac activity was observed (CRL:7.5mm). IUD wasn't observed within the uterine cavity, no signs of uterine rupture was noted either. the IUD was seen deep within transversalis fascia and abdominal cavity. However, it was noted that the device changed position too often and it shifted from right to left, this made IUD nearly impossible to be precisely located. According to the fact that the patient suffered from pain and was symptomatic the patient became a candid for laparoscopic surgery. Unfortunately, during the surgery, the device wasn't found and it was assumed that the initial ultrasound imaging was false and the device was still entrapped within myometrium. Another ultrasonography stated the device was actually within the abdominal cavity and it might be trapped inside omentum since it shifted as the patient changed position. since the first diagnostic evaluation couldn't locate and find the device it was decided that the device should be removed under ultrasonographic guidance. Prior to spinal anesthesia, the radiologist ran another ultrasonographic imaging using a superficial ultrasound probe. The location was then marked. (Figure 1) During the second laparotomy a paraumbilical incision was made and the device was found with the guidance and palpation as the device was barely visible and the threads were twisted inside the omentum. The foreign body had migrated into the omentum and was embedded into it. Granulation tissue was also formed around it which made it very difficult to distinguish. We performed partial omentectomy and the device was safely removed. (Figure 2 and 3) The patient was under observe for 24 hours, another ultrasonography assessed fetal cardiac activity and status. The patient was later discharged without any major complications.

(Figure1)

(Figure2)

(Figure3)

\section{Discussion}

Our case is 30-year-old woman who had an IUD implantation 2 months before admittance, a following work up due to IUD failure and pregnancy revealed that the device had perforated and penetrated abdominal cavity. Ultrasonographic imaging couldn't precisely locate the device since the device was fast shifting. The patient stated that she suffered from an unbearable pain which indicated that the device should be removed. During her first laparoscopic assessment we couldn't find and retrieve the device; therefore, tried using ultrasonographic guidance and it proved to be helpful and the surgery resulted in success. Even though the device was barely visible since it was embedded within the omentum, we removed it.

IUDs rarely perforate uterine but these few instances can possibly cause damage to internal organs. Several risk factors such as "inexperienced clinician, lactation, low parity and post-partum insertions particularly within 6 months after labor" are thought to be in association. Several cases of penetration into bowel and urinary tract have been reported. Patients with such perforations are prone to peritonitis. [5] [8] [4] [11]

Although several imaging modalities can locate the device the most preferred method in ultrasonography and as described by Rowlands et al the first alarming sign for perforation is missing threads and the back- 
bone of diagnosis is ultrasonography. [5]All IUD devices are radio opaque therefore they can be found on plain radiographs but this doesn't exactly reveal the position of device. A CT-scan can provide a more comprehensive view on the matter and in a few select cases the perforation was classified using this method. [5] [8] [16] [14]

Our patient was a pregnant woman therefore, we could solely use ultrasonography. We assume that since the device was cloaked with granulation tissue and omentum "which is quite loose and unrestricted" the position of the device couldn't be accurately found. Thus, more skill was required to locate the device and it could only be removed with ultrasonographic guide. During the surgery, the device was barely visible and could only be found with guidance and palpation.

The current consensus states that these devices should only be removed if the patient is symptomatic or there is a great risk of adhesions and complications such as perforation and peritonitis. Our patient was neither severely ill nor any signs of perforation was noted but since she was pregnant and prone to other complications and the pain was too great for her to bear, we decided to remove the device. [8] [9] [18] [14] $[12]$

Our experience with this patient has led us to presume that in patients whose foreign body can not accurately be positioned another imaging modality such as CT-scan must be utilized. If the patient has any contraindications, an ultrasonographic guide can be extremely helpful. In cases whose device is fast shifting and device is changing position too often, it must also be considered that the device might be lodged in tissues such as omentum that is loose and untethered; thereafter, a guidance can help with retrieving it. Our patients also posed another challenge which was due to its embedment within omentum this particular phenomenon indicates internal organs be thoroughly examined and palpated.

1. Thonneau P, Almont T, de La Rochebrochard E, Maria B. Risk factors for IUD failure: results of a large multicentre case-control study. Human Reproduction 2006;21 (10):2612-16

2. Thonneau P, Goulard H, Goyaux N. Risk factors for intrauterine device failure: a review. Contraception 2001;64 (1):33-37

3. Heinemann K, Reed S, Moehner S, Do Minh T. Risk of uterine perforation with levonorgestrel-releasing and copper intrauterine devices in the European Active Surveillance Study on Intrauterine Devices. Contraception 2015;91 (4):274-79

4. Caliskan E, Öztürk N, Dilbaz B, Dilbaz S. Analysis of risk factors associated with uterine perforation by intrauterine devices. The European Journal of Contraception \& Reproductive Health Care 2003;8 (3):150-55

5. Rowlands S, Oloto E, Horwell DH. Intrauterine devices and risk of uterine perforation: current perspectives. Open access journal of contraception 2016;7 :19

6. Teal SB, Sheeder J. IUD use in adolescent mothers: retention, failure and reasons for discontinuation. Contraception 2012;85 (3):270-74

7. Anderson SL, Borgelt LM. Case report: risk of uterine perforation from IUDs is greatest during postpartum period. American family physician 2013;88 (10):634

8. Zakin D, Stern WZ, Rosenblatt R. Complete and partial uterine perforation and embedding following insertion of intrauterine deives. I. Classification, complications, mechanism, incidence, and missing string. Obstetrical \& gynecological survey 1981;36 (7):335

9. Turok DK, Gurtcheff SE, Gibson K, Handley E, Simonsen S, Murphy PA. Operative management of intrauterine device complications: a case series report. Contraception 2010;82 (4):354-57

10. Aydogdu O, Pulat H. Asymptomatic far-migration of an intrauterine device into the abdominal cavity: A rare entity. Canadian Urological Association Journal 2012;6 (3):E134 
11. Farmer M, Webb A. Intrauterine device insertion-related complications: can they be predicted? BMJ Sexual \& Reproductive Health 2003;29 (4):227-31

12. Adoni A, Chetrit AB. The management of intrauterine devices following uterine perforation. Contraception 1991;43 (1):77-81

13. Boortz HE, Margolis DJ, Ragavendra N, Patel MK, Kadell BM. Migration of intrauterine devices: radiologic findings and implications for patient care. Radiographics 2012;32 (2):335-52

14. Kaislasuo J, Suhonen S, Gissler M, Lähteenmäki P, Heikinheimo O. Uterine perforation caused by intrauterine devices: clinical course and treatment. Human reproduction 2013;28 (6):1546-51

15. Heinberg EM, McCoy TW, Pasic R. The perforated intrauterine device: endoscopic retrieval. JSLS: Journal of the Society of Laparoendoscopic Surgeons 2008;12 (1):97

16. Cheung M-L, Rezai S, Jackman JM, et al. Retained intrauterine device (IUD): triple case report and review of the literature. Case reports in obstetrics and gynecology 2018;2018

17. Christodoulides AP, Karaolides T. Intravesical migration of an intrauterine device (IUD)-case report. Urology 2020;139:14-17

18. Tuncay Y, Tuncay E, Güzin K, Öztürk D, Omurcan C, Yücel N. Transuterine migration as a complication of intrauterine contraceptive devices: six case reports. The European Journal of Contraception \& Reproductive Health Care 2004;9 (3):194-200

\section{Declarations}

\section{Ethics approval and consent to participate}

This study was approved by the research and ethics committee of Tehran University of Medical Sciences. The patient's family have given their informed consent to publish this case.

\section{Consent for publication}

Written informed consent was obtained from the patient's next of kin for publication of this case report and any accompanying images. A copy of the written consent is available for review by the Editor-in-Chief of this journal.

\section{Competing interests}

The authors have no conflict of interest to declare.

\section{Funding}

None

\section{Authors' contribution}

A.T contributed in developing the research idea and composing and revising the manuscript. AH contributed in contributed in developing the research idea and composing and revising the manuscript. M.M contributed in composing and revising the manuscript. E.P contributed in composing and revising the manuscript.

\section{Acknowledgments}

None 


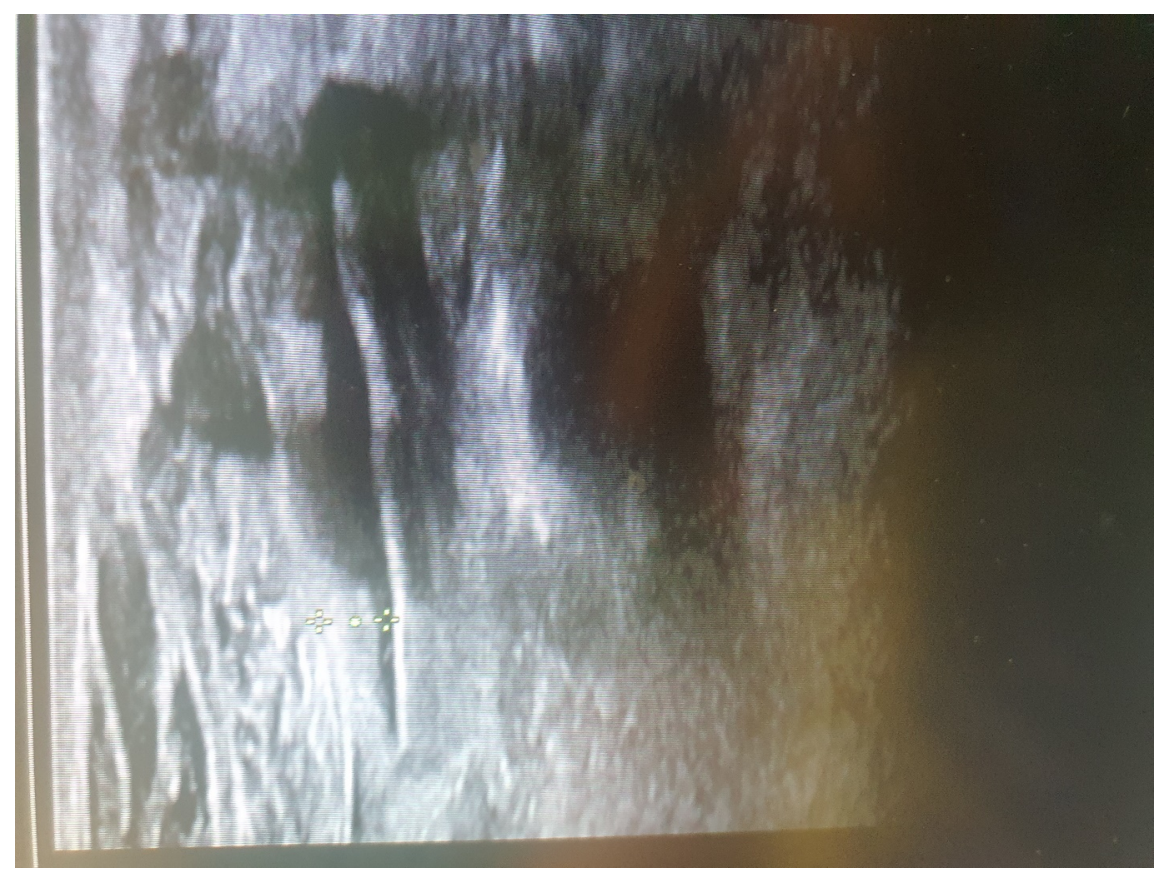

Figure 1. ultrasound imaging showcasing the device prior to surgery deep within abdominal cavity and omentum 


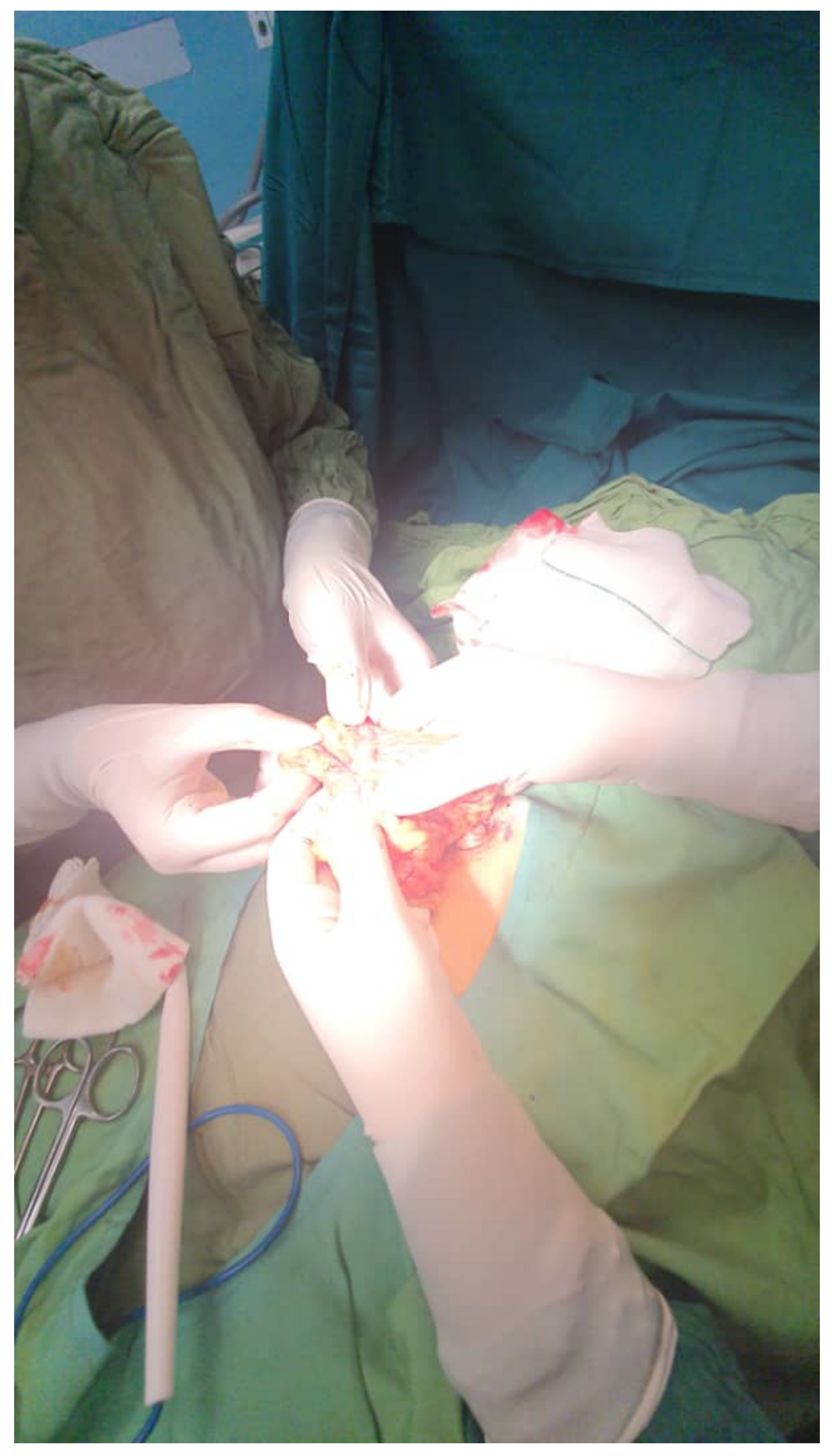

Figure 2. partial omentectomy 


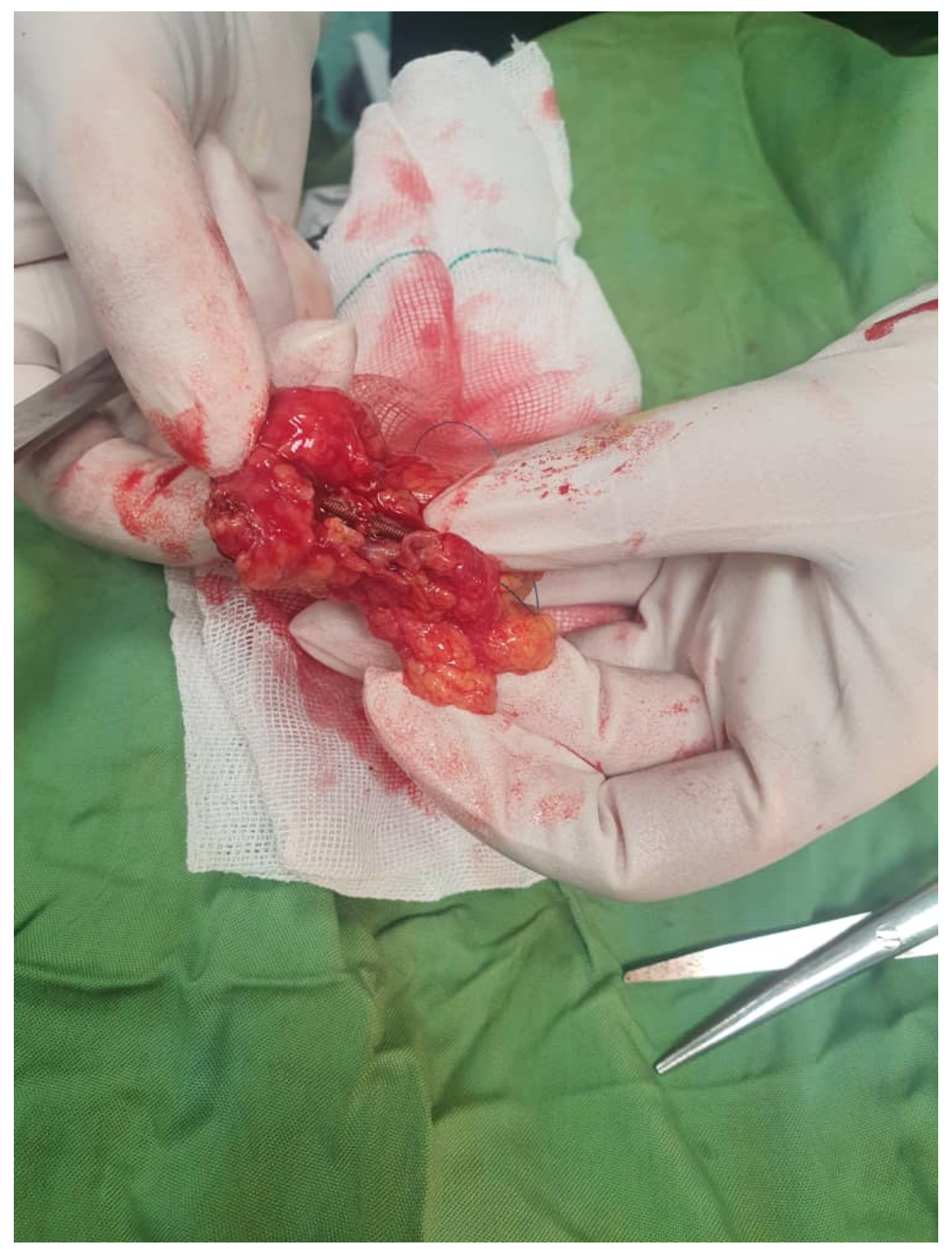

Figure 3. The part of omentum in which the IUD was embedded. Note that the threads had twisted inside and granulation tissue had surrounded the device 


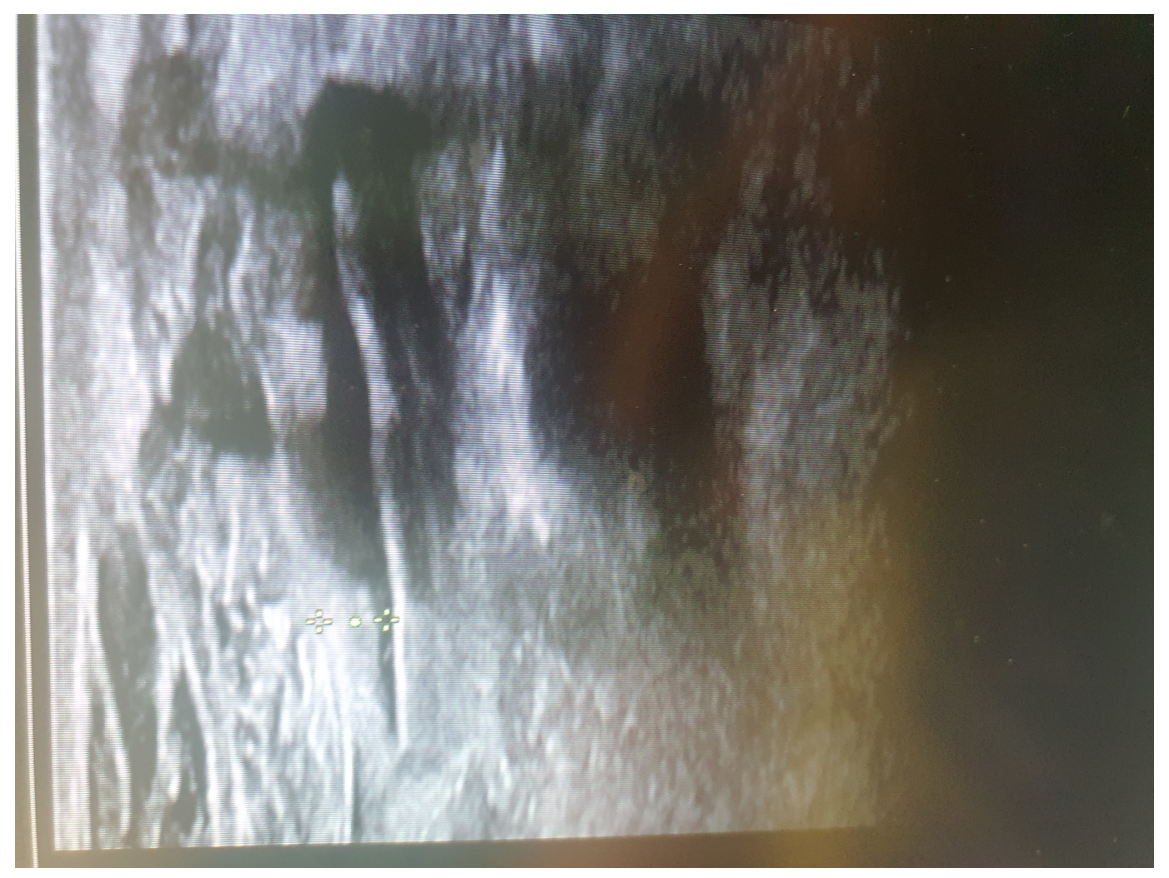




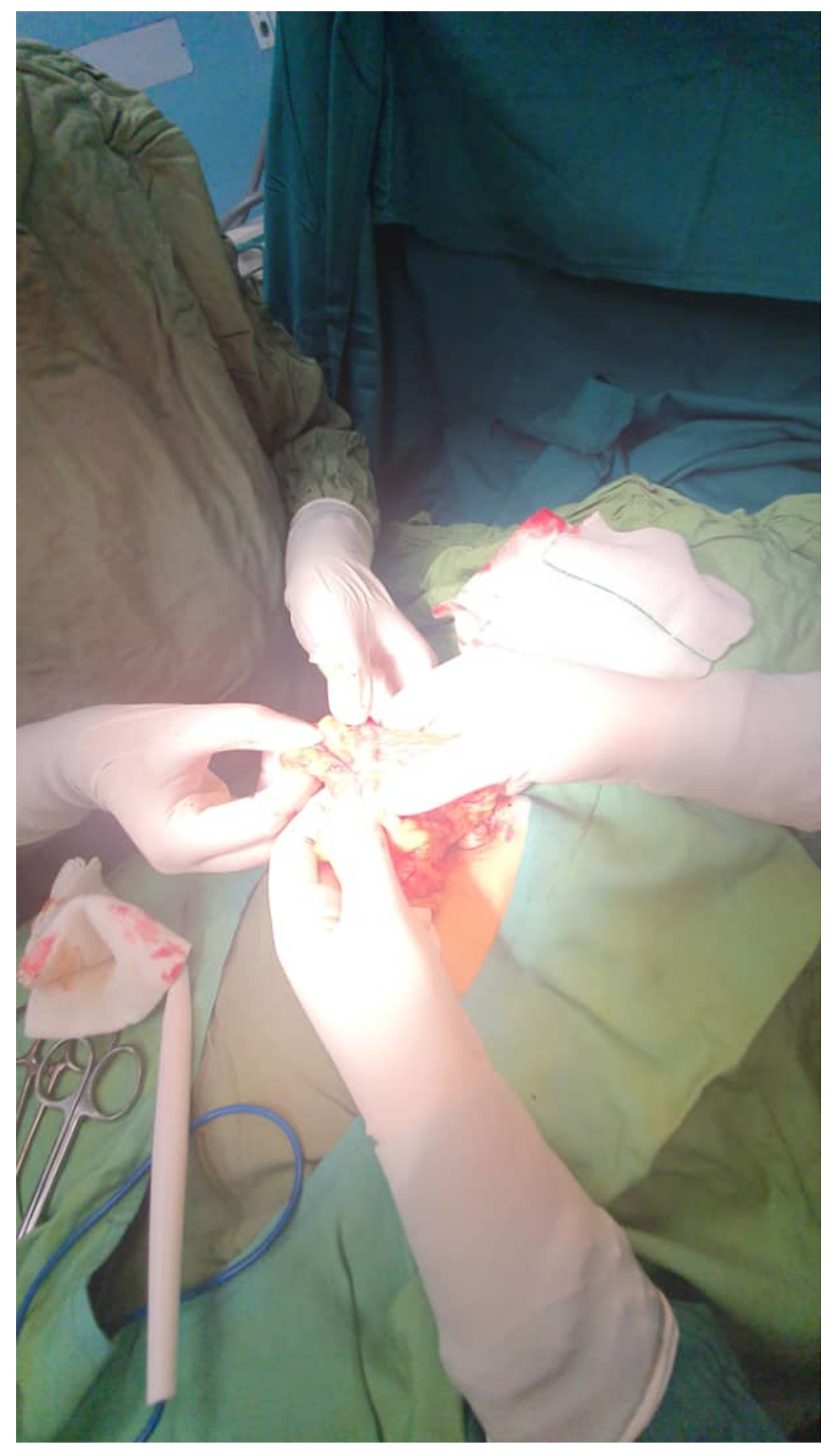




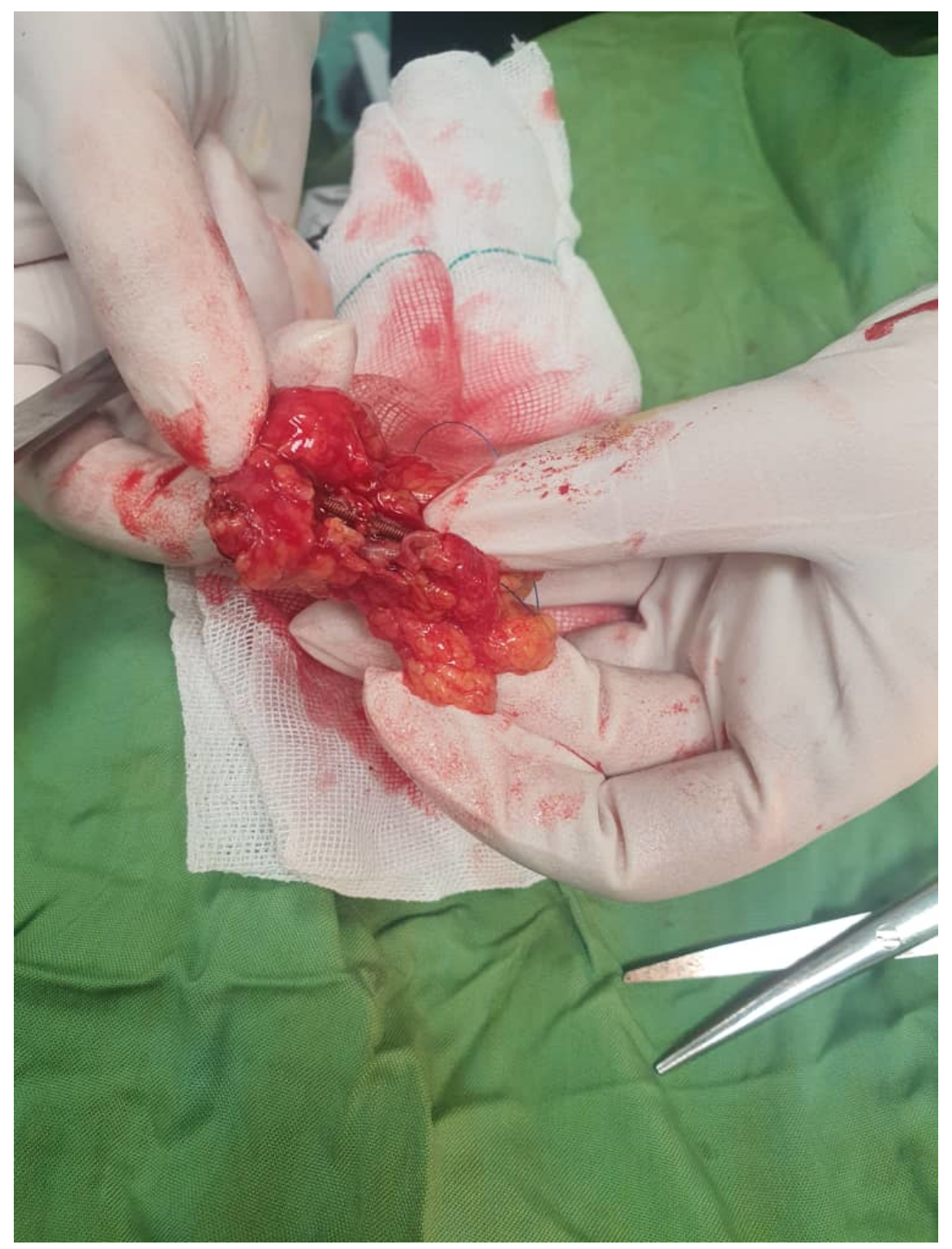

